\title{
Impact of Impurities and Crystal Defects on the Performance of CVD Diamond based Detectors
}

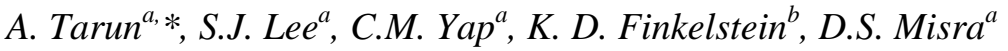 \\ ${ }^{a}$ IIa Technologies Pte Ltd. 17, Tukang Innovation Drive, 618300, Singapore. \\ ${ }^{b}$ Cornell High Energy Synchrotron Source, Cornell University, Ithaca, NY, 14853, USA. \\ *Corresponding author.Tel.: +65 6555 5825; E-mail address: atarun@2a-tech.com.sg
}

\begin{abstract}
Radiation detectors based on diamond are highly favored for particle physics research due to the superior radiation hardness. In this work, we investigate the influence of impurities and crystalline imperfections on the charge collection efficiency (CCE) of single crystal diamond. Seventeen ( 17 ) ultra-low fluorescent diamond samples grown by microwave plasma chemical vapor deposition method from IIa Technologies PTE LTD are pre-selected for this study. The measured CCE of all samples using ${ }^{241} \mathrm{Am}$ ( $\alpha$-particles) as ionizing source are analyzed together with the concentration of trace impurities and crystalline imperfection in the crystal. The amounts of impurities are quantified from integrated fluorescence intensity arising from the nitrogen vacancies (NV) created during different CVD growth process conditions. The crystal imperfections are assessed by X-ray rocking curves from X-ray topography images obtained at the Cornell High Energy Synchrotron Source. The CCE decays rapidly as the intensity of NV, $\left(I_{N V}\right)$, phonon sideband approaches that of diamond $2^{\text {nd }}$ order Raman peak which follows the relation: $\operatorname{CCE}\left(I_{N V}\right)=100 /\left(1+\left(\frac{I_{N V}}{1.0}\right)^{\frac{5}{2}}\right)$. The energy resolution, $\Delta \mathrm{E} / \mathrm{E}$, (ratio of the energy spectrum width to the most probable peak) highly correlates with broader rocking curve width distribution.
\end{abstract}

\section{Introduction}

Diamond has attracted attention as an ideal material for radiation detection due to its remarkable intrinsic properties ${ }^{1-6}$. The intrinsic wide band gap of $5.5 \mathrm{eV}$ of the diamond creates exceptionally low number of thermally-excited free charge carriers resulting to a very low leakage current during operation. ${ }^{2-4}$ The excellent radiation hardness of diamond enables operational-stability in harsh radiation environment without significant changes in the signal integrity. Additionally, the high charge carrier mobility provides fast and large signal pulse in particle detection ${ }^{4-5}$. Recent progress in diamond purity and crystalline quality of single crystal diamond (SCD) grown by microwave plasma assisted chemical vapor deposition (MPCVD) method have improved to the extent that these imperfections do not prohibit their use in diamond based sensors $^{2-5}$ such as $\mathrm{x}-$-ray $^{6-7}$ and particle detectors. ${ }^{4-5}$ However, it has been shown ${ }^{8}$ that nitrogen impurities exceeding $1 \mathrm{ppb}$ as measured by electron paramagnetic resonance (EPR) deteriorates the charge carrier mobilities and lifetimes of diamond based detectors. These infinitesimal nitrogen impurities which degrade the performance of the device are still present in CVD diamonds and will continue to be a concern for their use in radiation detection. An in-depth analysis on the electronic impact of nitrogen related impurities are therefore equally important as lattice perfection to obtain high quality diamonds. Understanding the most important factors that contribute to degradation of charge collection efficiency (CCE) and developing an 
efficient, in-expensive and non-destructive technique to provide reasonable estimation of CCE is therefore of great importance. In this work, we combined Raman/fluorescence (R/FL) spectroscopy and high resolution X-ray topography to systematically investigate the effect nitrogen related impurities and crystal lattice imperfections, respectively, on the CCE of electronic-grade diamonds that can be used as particle detection devices. We discuss the electronic impact of increasing fluorescence intensity on the measured CCE. The variation in fluorescence intensity depends on the concentration of nitrogen impurities incorporated during CVD growth process. We visualized crystal defects such as thread dislocations and crystal plane twining and study their effects on the measured CCE.

\section{Experimental Details}

The (001)-oriented single crystal diamond plates used in this study were fabricated by IIa Technologies Pte Ltd. The samples were mechanically polished down to approximately $400 \mu \mathrm{m}$ thickness. Fourteen (14) diamond plates with varying level of impurities (i.e. nitrogen) were carefully selected based on the measured R/FL spectra. The spectra were collected at room temperature and at a fixed power and exposure time using the in-Via Raman microscope (Renishaw) with $514.5 \mathrm{~nm}$ laser excitation focused by $50 \mathrm{X}$ objective lens. For each diamond plate sample, $12 \mathrm{R} / \mathrm{FL}$ spectra were collected that is 6 measurement spots on the top and bottom sides. It is worth mentioning that no significant variations were observed on the 12 spectra which suggest that impurities are uniformly distributed across the sample. The incremental spatial variation in the fluorescence intensity across the sample is much lower than the fluorescence intensity variation from sample to sample. Figure 1 overlays the representative R/FL spectra of all samples after normalization to the first order Raman peak intensity of diamond at $552.37 \mathrm{~nm}\left(1 \times 10^{7} /\left[\left(1 \times 10^{7} / 514.5 \mathrm{~nm}\right)\right.\right.$ $\left.1332.5 \mathrm{~cm}^{-1}\right]$ ). We can clearly see that the impurity-induced fluorescence between 600 to $900 \mathrm{~nm}$ with the exception of $\mathbf{S 1 4}$ is very weak and that the NV related impurities are almost negligible. Focusing on the weak spectral features between 550 to $850 \mathrm{~nm}$ for $\mathbf{S 1}$ to $\mathbf{S 1 3}$ (see Figure 1, inset) reveals the well-defined $2^{\text {nd }}$ order Raman mode of diamond centered at 598nm. This validates the improved quality of diamonds. Due to the very weak signal from the $\mathrm{NV}$ defects, $\mathrm{NV}^{0}(575 \mathrm{~nm})$ and $\mathrm{NV}^{-}(638 \mathrm{~nm})$, the integrated intensity of the corresponding phonon side band has been selected as a more reliable measure of impurities. ${ }^{9}$ The inset of Figure 1 shows the broad phonon side band of the NV defects of varying integrated intensity $\left(\boldsymbol{I}_{\mathbf{N V}}\right)$ between $600 \mathrm{~nm}$ to $850 \mathrm{~nm}$. 


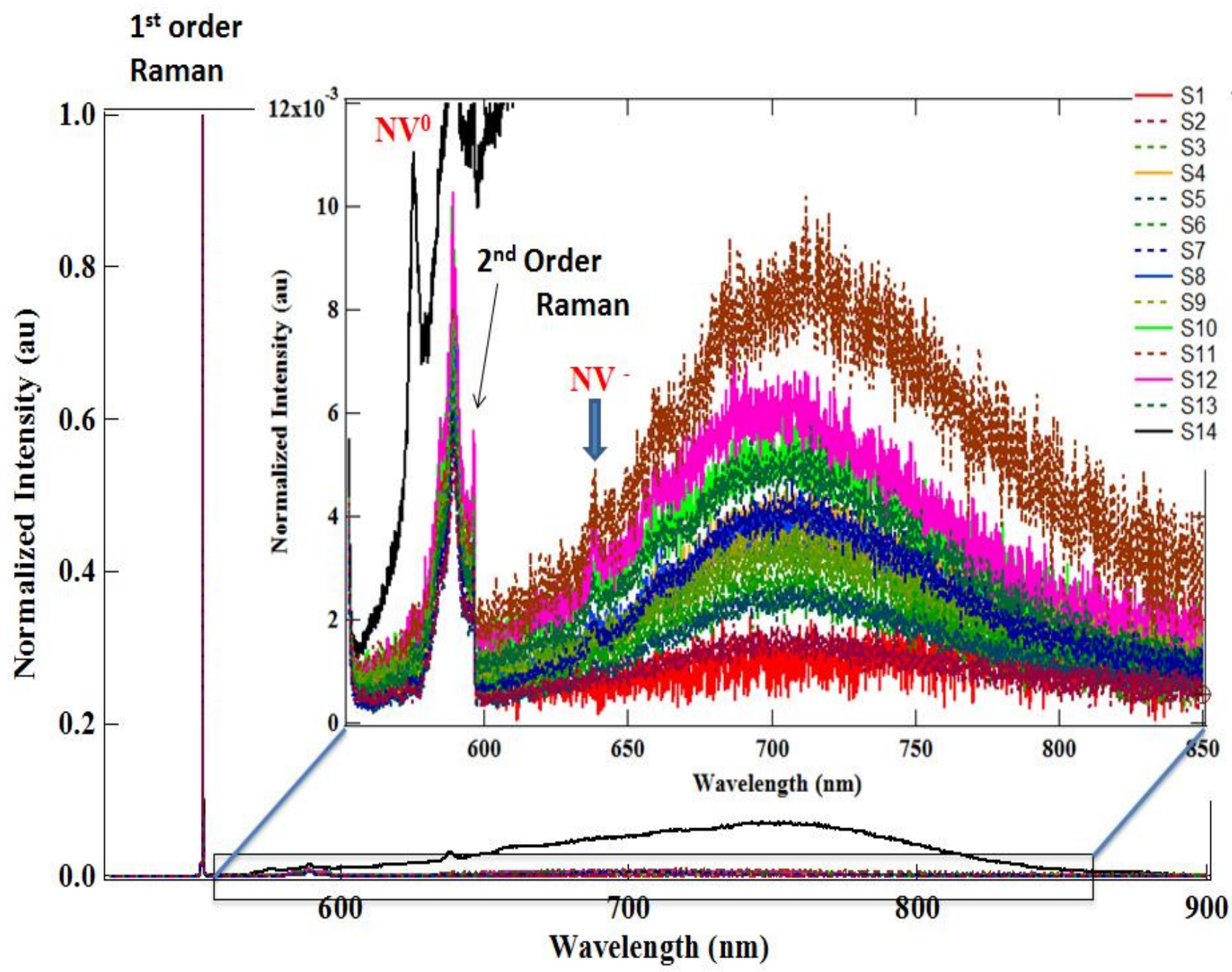

Figure 1. Averaged Raman/ Fluorescence spectra of the 14 diamond plates. Inset: zoomed plots showing the broad phonon side band of NVs.

To generate ohmic contacts, the samples were exposed to oxygen plasma to form oxygen-terminated surface. The samples were further boiled in acid solution $\left(\mathrm{H}_{2} \mathrm{SO}_{4}+\mathrm{HNO}_{3}\right)$ not only to ensure full surface oxidation but also to remove non-diamond and residual impurities introduced during polishing. A thin layer of $\mathrm{Cr} / \mathrm{Au}(50 \mathrm{~nm} / 50 \mathrm{~nm})$ electrodes were sputtered on both sides of the diamond samples, forming a sandwich parallel plate detector structure. Sputtering is performed with in-situ annealing at $150^{\circ} \mathrm{C}$ in argon atmosphere. Prior to CCE measurement, current - voltage (I-V) measurements were performed on all samples using high precision Keithley electrometer to determine the detector safe operating region. I-V measurements will also ensure that ohmic contacts are fabricated and not the formation of Schottky barrier that arises as a consequence of a mismatch between the semiconductor and metal Fermi levels which depends on the metal work function $^{10-11}$.

The CCE measurement was carried out using the setup showed in Figure 2a. The diamond sample is irradiated with collimated $5.48 \mathrm{MeV} \alpha$-particles in vacuum $\left(10^{-3} \mathrm{mbarr}\right)$ from the ground electrode (GND). At this alpha energy, the ionization can only take place within the top $12 \mu \mathrm{m}$ layer of diamond sample. ${ }^{10}$ The detection process relies on the induced current in external circuits due to the motion of radiation-generated electron-hole pairs when alpha particle impinges on the diamond. The external circuit is composed of charge sensitive preamplifier which accepts detector bias from high voltage (HV) supply and applies it to detector through the amplifier input. The bias voltage creates a uniform electric field $(\boldsymbol{E})$ within the diamond in which electrons and holes drift apart. The induced current in external circuit is amplified by a charge sensitive 
preamplifier and by a linear shaping amplifier (with a shaping time of $0.5 \mu \mathrm{s}$ ). The signal is then fed to a multichannel analyzer for pulse height analysis. We would like to emphasize that careful measurement design must be taken to eliminate the accumulation of space charge and polarization ${ }^{10-11}$.

Figure $2 \mathrm{~b}$ shows the collected CCE spectrum for sample $\mathbf{S 1}$ after 20 hours of irradiation. The abscissa in Figure $2 \mathrm{~b}$ is calibrated with a pulser using $12.85 \mathrm{eV}$ as the energy required to create an electron-hole pair in diamond ${ }^{11}$. The CCE $=99.1 \%$ of $\mathbf{S 1}$ is calculated by fitting the obtained energy spectrum with the negative landau distribution to obtain the most probably peak. This measurement is performed on all 14 samples.

(a)

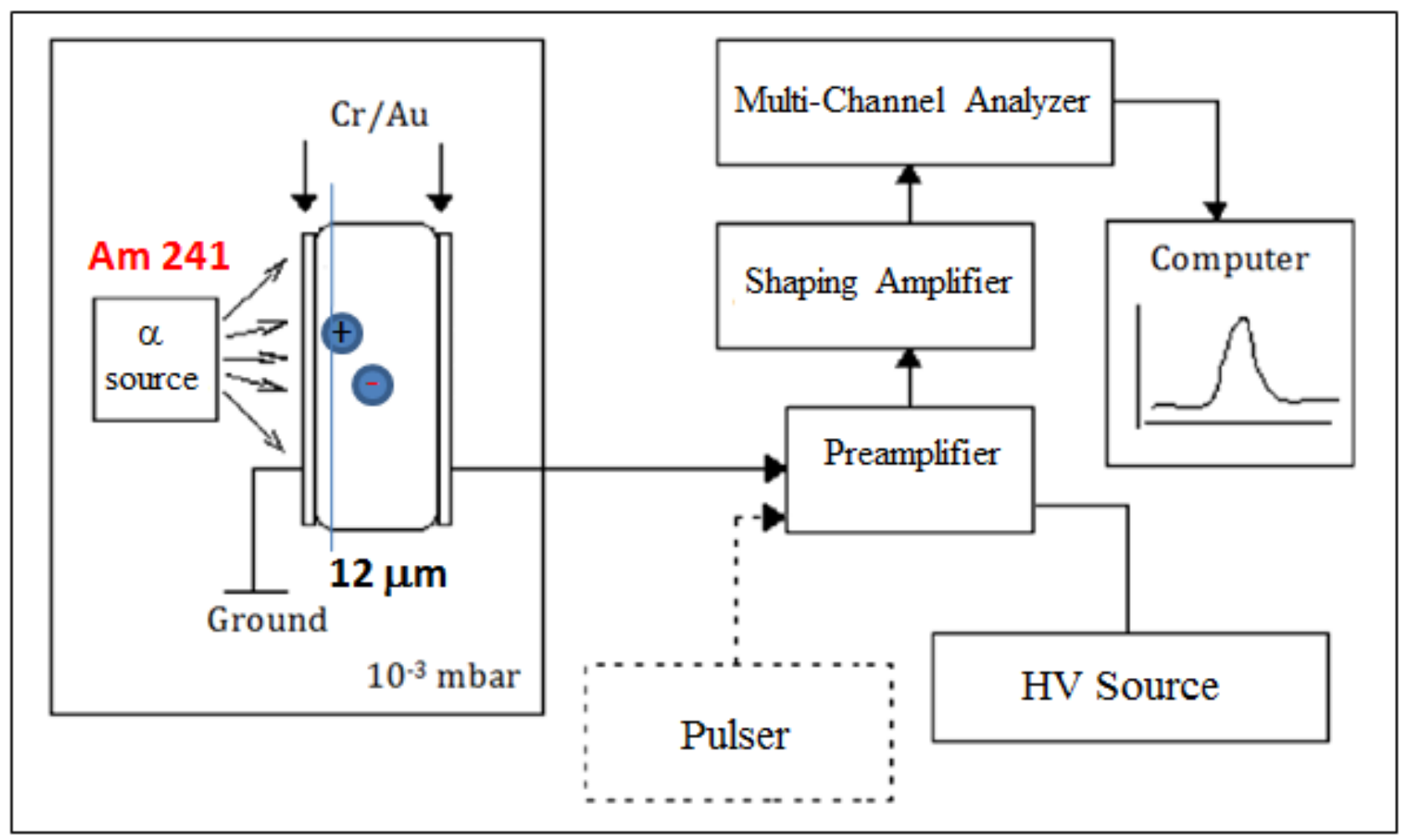

(b)

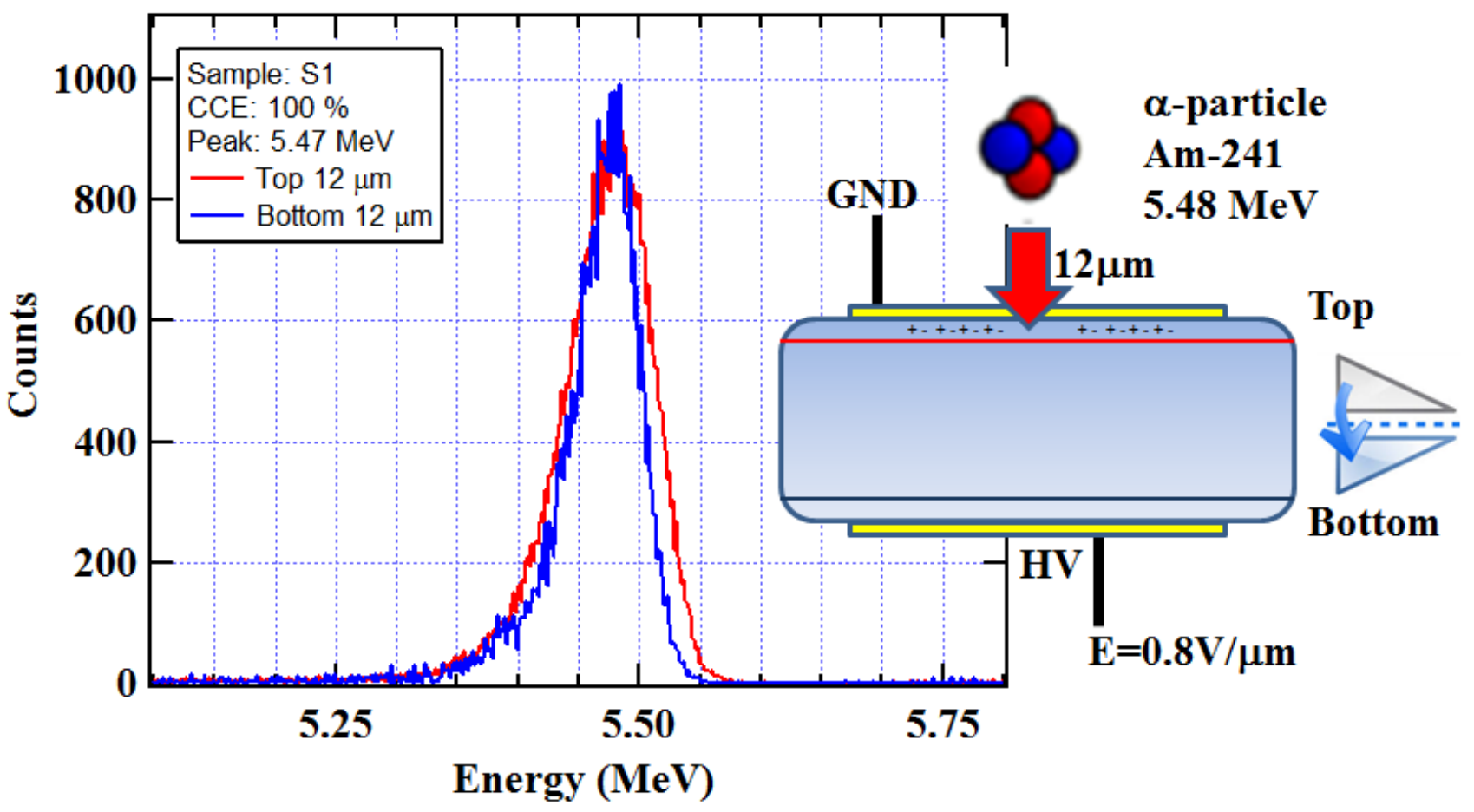

Figure 2. (a) Schematics of measurement setup and signal detection process. (b) A typical CCE spectrum obtained from top and bottom $12 \mu \mathrm{m}$ of the thick diamond plate. 


\section{Results and discussion}

The CCE for each sample was then plotted against their corresponding $\boldsymbol{I}_{N V}$ as shown in Figure 3. The CCE drops rapidly as the intensity of NV phonon sideband approaches that of diamond $2^{\text {nd }}$ order Raman peak, and reaches a plateau when the two levels of intensities are comparable. In order to accurately describe the behaviour of the rapid decrease in the CCE as a function of impurities in the diamond bulk, the data is fitted using the equation below which exhibits the following relation:

$$
\operatorname{CCE}\left(I_{N V}\right)=\frac{C C E_{\max }}{1+\left(\frac{I_{N V}}{X_{1 / 2}}\right)^{\text {Rate }}}
$$

where, $\boldsymbol{I}_{N V}$ is the integrated intensity of the broad phonon side of the NV defects between $600 \mathrm{~nm}$ to $850 \mathrm{~nm}$ in the diamond. It provides a qualitatively estimate of the concentration of nitrogen impurities. $\boldsymbol{X}_{1 / 2}$ represents the overall sensitivity and noise of the measuring system and will have a similar unit of measurement as $\boldsymbol{I}_{N V}$. It tells us at which $\boldsymbol{I}_{N V}$ value will give rise to $50 \% \mathrm{CCE}$. $C C E_{\max }$ is the maximum CCE when the impurity approaches zero $\left(I_{N V} \rightarrow 0\right)$. Rate describes how rapidly the CCE drops with $\boldsymbol{I}_{N V}$. Although, $\boldsymbol{I}_{N V}$ is expressed in arbitrary units, it provides a good representation of the total amount of impurity present in relation to the CCE of the CVD diamond bulk. Using the modified rate equation above to perform curve fitting on the experimental data (see Figure 3), we found that the best we fit value for the Rate is 2.5 and $\boldsymbol{X}_{1 / 2}$ is 1.0. It implies that the CCE decays rapidly with $\boldsymbol{I}_{\boldsymbol{N} V}$. The rate of decay is 2.5 . The error bars along the $x$-axis of roughly 10\% shown in Figure 3 were attributed to both the system noise and measured intensity fluctuations at 12 different positions in the sample. We would like to point out that for samples having extremely low or "flat" fluorescence intensity, i.e., S1 and S2 in Figure 1, the accuracy of the obtained integrated intensity $\boldsymbol{I}_{N V}$ is lesser and dominated by system noise. This is due to the limited detection sensitivity the system. Measurement at cryogenic temperature may be an option to enhance the measurement sensitivity level aside from using sophisticated low noise charge coupled device camera to demonstrate accurate CCE dependency beyond 90\%. The error bars along the $y$-axis are estimated from the standard deviation $( \pm 3 \sigma)$ of the three separate CCE measurements under the same bias conditions.

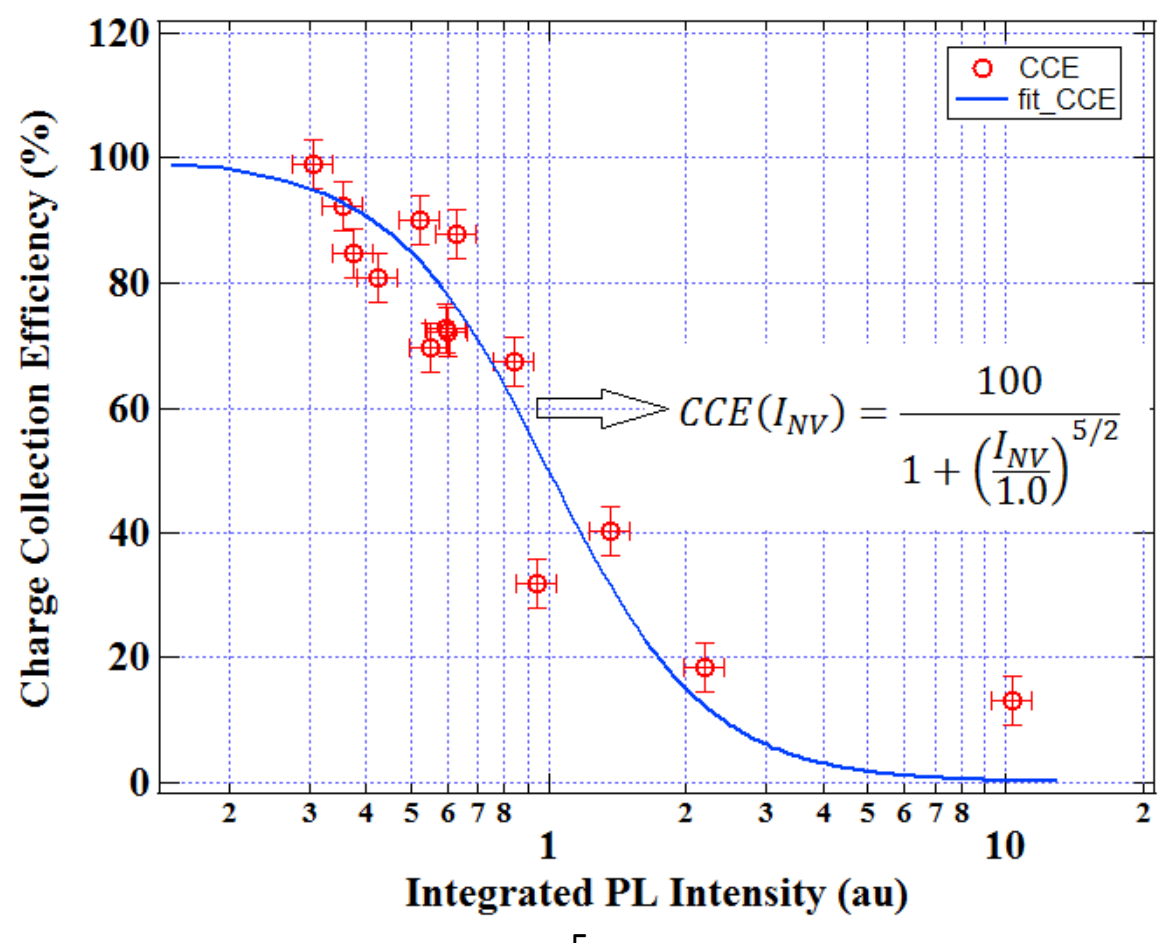

Figure 3. Plot of CCE as a function of integrated NV phonon side band. Solid line is the fit used to describe the rapid decay of CCE with $I_{N V}$. 
Aside from nitrogen vacancies, lattice defects and dislocations are known to deteriorate the CCE by trapping the charge carriers and reducing their lifetimes ${ }^{8}$. These electrically active crystal imperfections in the whole sample area are efficiently mapped in 2D using the high energy single wavelength X-ray beam (C1 beamline) at Cornell High Energy Synchrotron Source (CHESS). The single wavelength was acquired from silicon (331) monochromator whose $d$-spacing closely matches the diamond (220) reflection, where we set the diamond plate to diffract using those lattice planes in a vertical scattering geometry. X-ray rocking curve (RC) imaging were obtained from a series of 2D X-ray intensity images from (220) diamond crystal plane recorded at each setting of the Bragg angle ${ }^{7}$. This whole process is repeated for each sample. The Xray intensity at a particular pixel location from series (varying Bragg angle) of X-ray images was tabulated and fitted with Gaussian function to determine the full width at half maximum (FWHM). For every pixel, the extracted FWHM of the RC are mapped to their original pixel location to construct the RC width image as shown in Figure $4(\mathrm{a}-\mathrm{c})$. Three (3) samples were characterized and plotted with same FWHM range for comparison. The corresponding distribution of the RC width is displayed as histogram in Figure 4 (d-f). Generally, a smaller RC width (darker regions) indicates good lattice structure and a larger RC width (brighter region) indicates lattice imperfection. Sample $\mathbf{S 1}$ have the lowest average RC width of $15 \mu$ Radians and nearly the same throughout the whole sample area which can also be clearly seen from the very narrow histogram (see Fig. 4d). We found that the CCE for this sample is 99.1\%. For sample S5 (Fig. 4b) and S11 (Fig 4c), the average RC widths are higher with broader histogram which suggests that they have more lattice disruptions. Not surprising, samples $\mathbf{S 5}=84.7 \%$ and $\mathbf{S 1 1}=40.3 \%$ shows lower CCE compared to $\mathrm{S} 1$ $=99.1 \%$. Clearly, the observed decrease in CCE in S5 and $\mathbf{S 1 1}$ can be attributed to both an increase in fluorescence intensity and broadening and increase in RC width histogram. It is not substantial to conclude at this point whether the increase in fluorescence is the main origin of the decrease in CCE. This is because the degradation of the RC might also be due to increase in the fluorescence impurities in the sample which contributes to the disruptions in the lattice. It is worthwhile to note that a poor RC can also be due to effects not related to impurities such as some plastic (or even elastic) deformation of the lattice. Besides, for diamond plates having higher levels of nitrogen impurities (i.e. HPHT grown diamonds), ${ }^{12}$ the CCE is very low even though the crystal perfection is very good. Hence, the effect of lattice imperfection to the CCE has so far remained elusive at this point. 
Map of X-ray rocking curve width

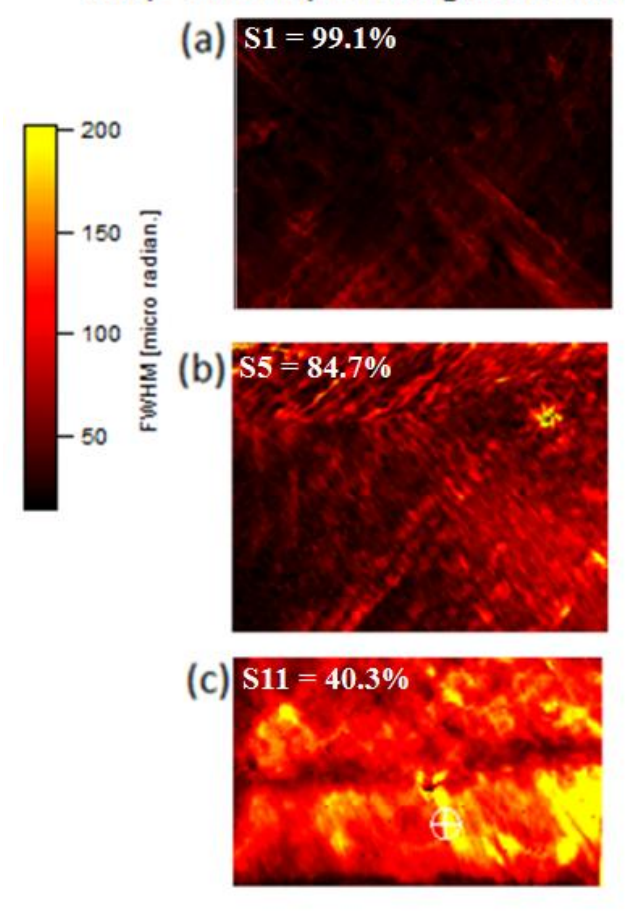

Histogram of Rocking Curving

(d)

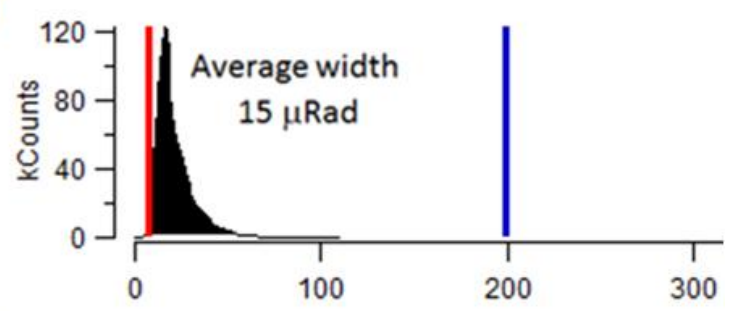

(e)
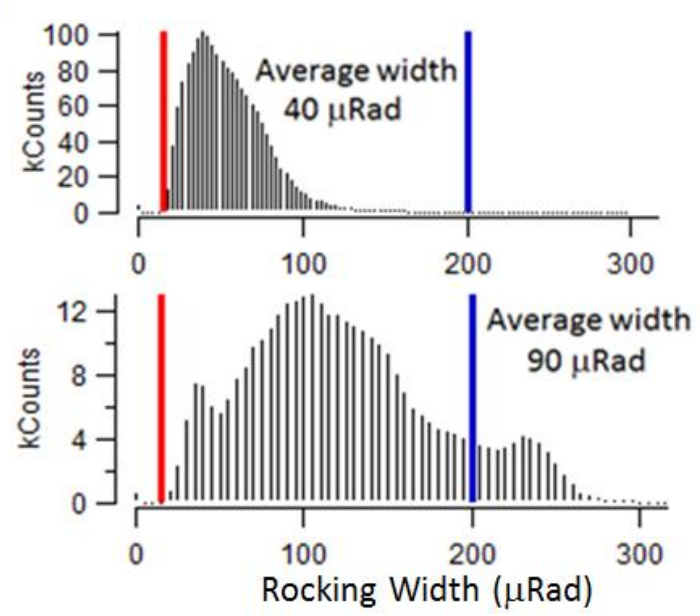

Figure 4. Map of X-ray rocking curve width and respective histogram.

In order to determine the effect of lattice defects or distortion onto the CCE, we selected additional three (3) samples of nearly the same impurity level but of different rocking width. Figure 5 shows fluorescence spectra of the 3 samples having nearly the same level of fluorescence intensity along with S1 for comparison. The inset shows the ${ }^{241} \mathrm{Am}$ energy spectra obtained from these samples at $0.8 \mathrm{~V} / \mu \mathrm{m}$ bias field. The data was fitted with negative landau distribution, $f(x)=\operatorname{Aexp}\left(\frac{-1}{2}\left\{\frac{-x+x_{0}}{B}+e^{-\left(\frac{-x+x_{0}}{B}\right)}\right\}\right)$, to obtain the most probably peak $\left(x_{0}\right)$ for CCE calculation. The width of the spectrum provides the energy resolution response of the diamond detector which can be obtained from $B$. The fitting results were tabulated in Table 1 and also depicted at the inset and labeled FWHM/Width. From the inset, although the fluorescence (impurity) levels are of the same intensity we still found a slight decrease $(2.2 \%)$ in the CCE. For example, S17 which is found to have lower fluorescence compared to $\mathbf{S 1 5}$ and $\mathbf{S 1}$ shows lower CCE = $5.36 / 5.48=97.8 \%$. Figure 6 shows the map of $\mathrm{x}$-ray RC width of the three samples accompanied by their respective histogram. Sample $\mathbf{S 1 7}$ which has broader distribution of RC width and higher most probable peak in the RC width histogram shows a drop in the CCE even though it has the lowest fluorescence intensity among the three. It is also interesting to note that the obtained energy spectrum broadens with broader $\mathrm{RC}$ width distribution. The energy resolution, $\Delta \mathrm{E} / \mathrm{E}$, defined as the ratio of the energy spectrum width to that of the most probable peak are listed in Fig. 6. We observed that reduced energy resolution of the detector is highly correlated with increased RC width. This can be attributed to the fact that a disruption in the lattice or lattice strain affects the mean drift velocities of both holes and electrons thereby broadening the energy spectrum and also slightly reducing the CCE (lower energy resolution, $\Delta \mathrm{E} / \mathrm{E}$ ). Further experimentation is needed to know if there is a correlation between reduced CCE and to either lattice distortion or lattice strain. Based on this result, we concluded that the significant drop in the CCE in Figure 4 is predominantly attributed to the decreasing impurity levels from S1, S5 and S11. The contribution from the intensification of inherent lattice defects (broadening and increase of RC width distribution) is marginal relative to increasing fluorescence intensity.

Table 1. Detector performance summary obtained from energy spectrum and RC width histogram 


\begin{tabular}{|c|c|c|c|c|}
\hline Sample ID & CCE Peak (\%) & $\begin{array}{c}\text { CCE Width } \\
(\mathrm{MeV})\end{array}$ & $\begin{array}{c}\text { RC Width } \\
(\mu \text { Radian })\end{array}$ & $\begin{array}{c}\text { Peak of RC Width } \\
\text { Distribution( } \mu \text { Radian })\end{array}$ \\
\hline S15 & 100 & 0.04 & 3.43 & 10.12 \\
\hline S16 & 99.1 & 0.16 & 5.8 & 10.15 \\
\hline S17 & 97.8 & 0.16 & 15.63 & 16.01 \\
\hline
\end{tabular}

Clearly, the determination of the RC width on the entire sample can be a useful method to further assess the electronic properties (i.e. CCE) but only for diamond with already very low amount of impurities. Both an ultra-low fluorescence and narrow $\mathrm{RC}$ width across the entire samples are important in making a good detector based on diamond.

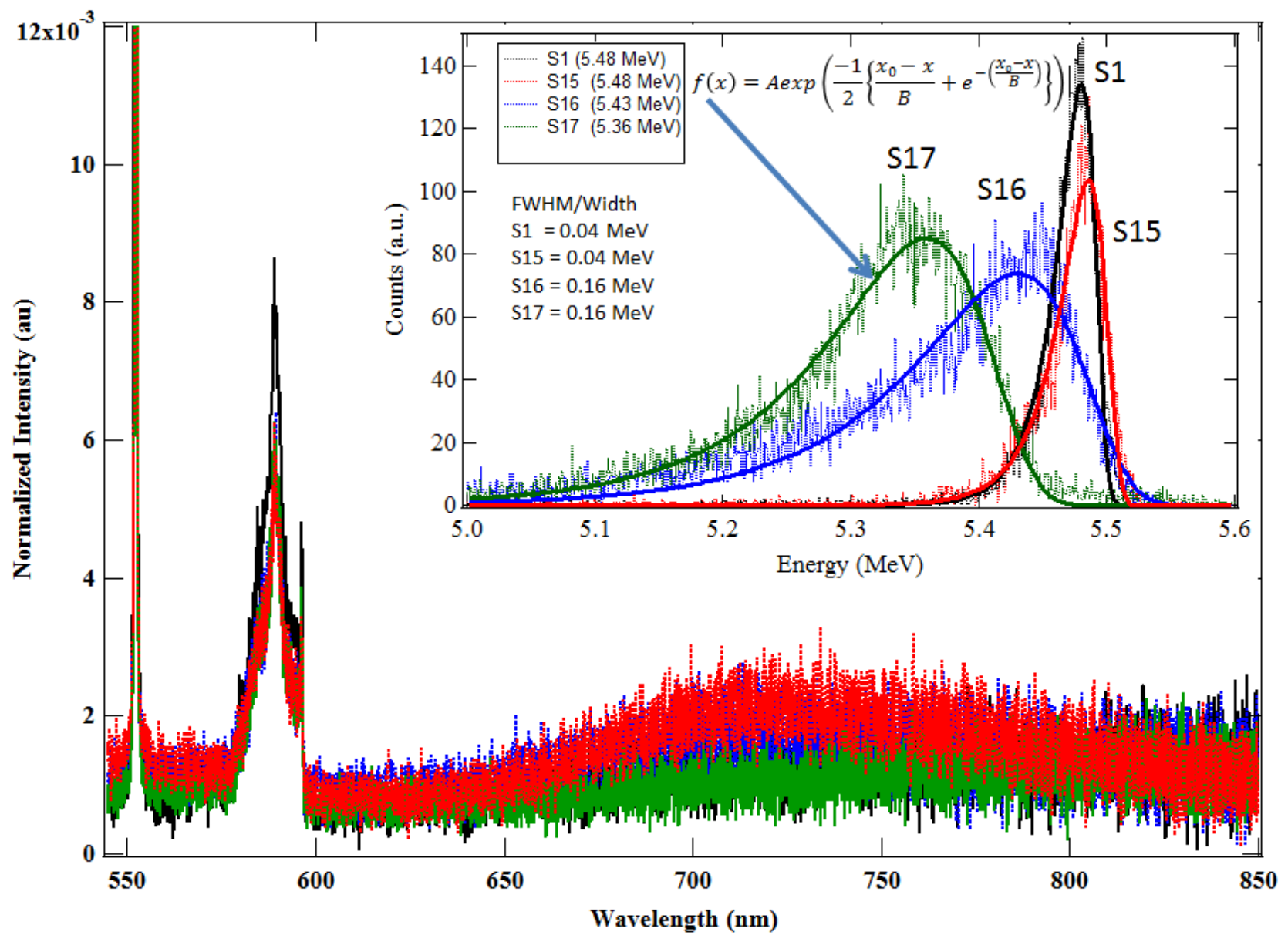

Figure 5. Fluorescence spectra of diamonds for energy resolution dependence study. Inset: Energy spectrum obtained from ${ }^{241} \mathrm{Am}$ source using a diamond detector with a bias field of $0.8 \mathrm{~V} / \mu \mathrm{m}$ 

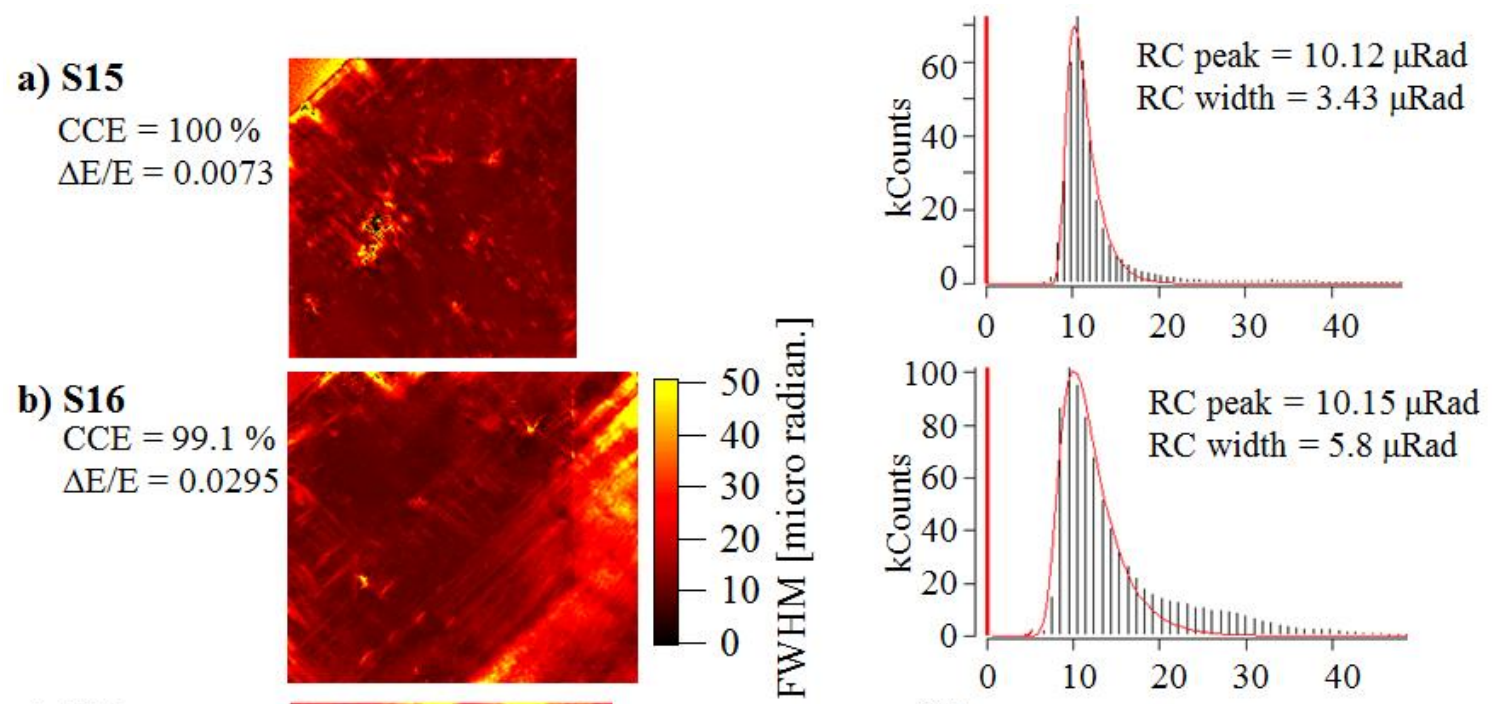

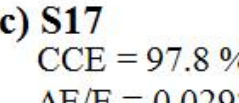
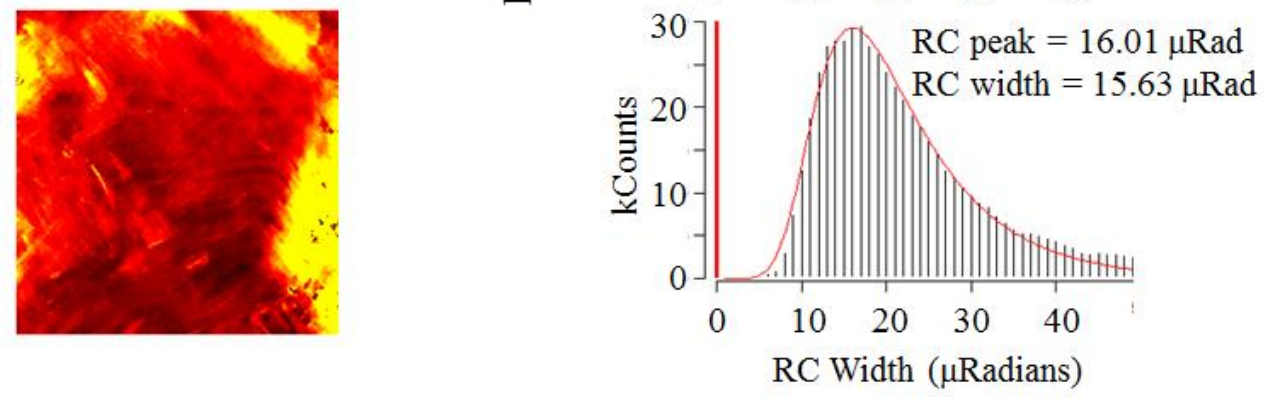

Figure 6. Map of the rocking curve width of diamond detectors. The CCE and the energy resolution, $\Delta \mathrm{E} / \mathrm{E}$, obtained from the measured energy spectrum shown in Figure 5 were depicted. For quantification of the most probable RC peak and RC width, the RC width histogram corresponding to each sample is also shown on the right.

\section{Summary}

Our work has established that the CCE of diamond decays exponentially as the integrated fluorescence intensity of NV phonon sideband increases. When the intensity level of the NV phonon sideband approaches that of the $2^{\text {nd }}$ order Raman intensity, the CCE starts to plateau. The CCE of diamond were also found to be slightly affected by the lattice defects. The energy resolution of diamond-based detectors highly correlates with broadening and increase of RC width distribution. We have successfully demonstrated two nondestructive methods to provide better insights on the charge collection efficiency of electronic grade diamond plates for applications in diamond based detectors and sensors.

\section{Acknowledgement}

The X-ray rocking curve measurement is conducted at the Cornell High Energy Synchrotron Source (CHESS) which is supported by the National Science Foundation and the National Institutes of Health/National Institute of General Medical Sciences under NSF award DMR-1332208. We also would like to acknowledge Prof Robert Stone of Rutgers University for his advices on the CCE measurements. 


\section{References}

[1] S. Wang, P. Sellin, A. Lohstroh. Carbon 43 (2005) 3167-3171.

[2] C. E. Nebel and M. Stutzmann. Electronic Materials Information Service, Vol. 26, Chap A1.6, p. 40.

[3] D. Meier et al., Nucl. Instrum. Methods Phys. Res. A 426 (1999) 173.

[4] J. Isberg et al., Science vol. 297 (2002) 1670-1672.

[5] D. Dobos and H. Pernegger, Nucl. Instrum. Methods Phys. Res. 246 (2011) A 628(1).

[6] J. W. Keister, J. Smedley, E. M. Muller, J. Bohon, and A. Heroux, Nucl. Instrum. Methods Phys. Res. A 649 (2011) 91-93.

[7] G.L. Yang, R. Jones, F. Klein, K. Finkelstein and K. Livingston, Diam. Relat. Mater. 19 (7-9) (2010) 719-722.

[8] A. Secroun, O. Brinza, A. Tardieu, J. Achard, F. Silva, X. Bonnin, K. De Corte, A. Anthonis, M.E. Newton, J. Ristein, P. Geithner, A. Gicquel. Physica Status Solidi (a) 204, No. 12. (2007) 4298-4304.

[9] T. L. Wee, Y. K. Tzeng, C. C. Han, H. C. Chang, W. Fann, J. H. Hsu, K. M. Chen, Y. C. Yu. J. Physics. Chem. A (2007), 111, 9379-9386.

[10] M. Pomorski, E. Bedermann, W.de Boer, A. Furgeri, C. Sander, J. Morse. Diam. Relat. Mater. 16 (2007) 1066-1069.

[11] A. Galbiati, S. Lynn, K. Oliver, F. Schirru, T. Nowak, B. Marczewska, J. A. Duenas, R. Berjillos, I. Martel, L. Lavergne. IEEE Transactions On Nuclear Science, Vol. 56, No. 4 (2009) 1863-1874.

[12] L. M. Reinitz, P. R. Buerki, J. E. Shigley, S. F. McClure, T. M. Moses. G\&G 36 (2) (2000) 128-137. 


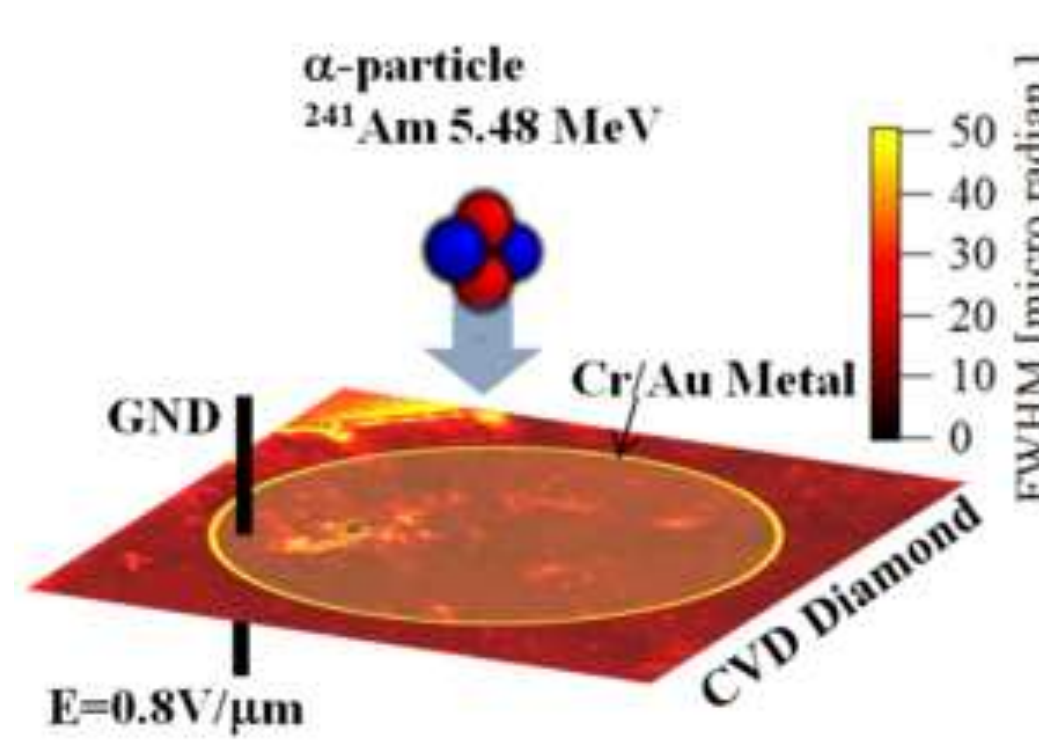

Diamond Radiation Detector

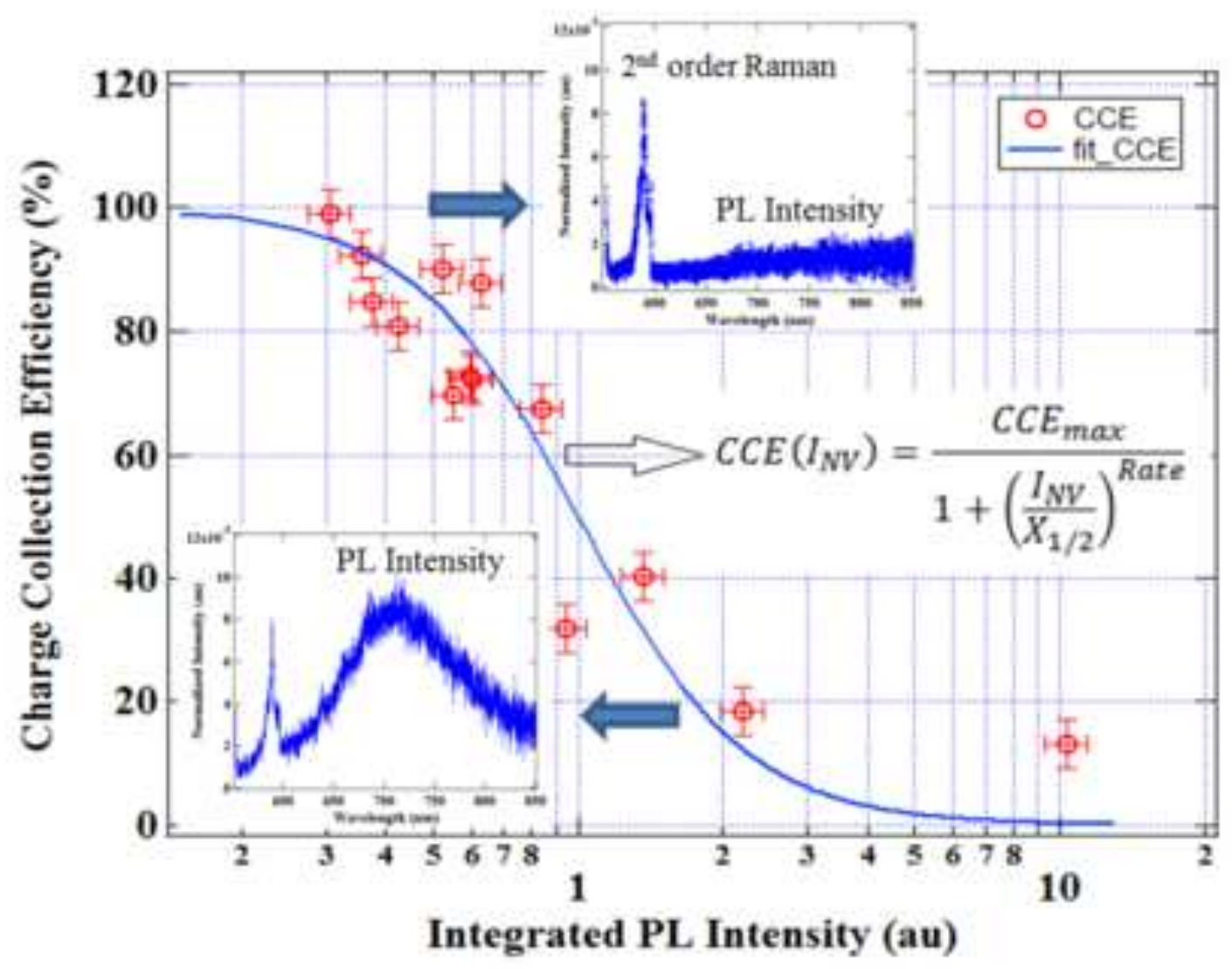

